\title{
APPLYING THE ABDUCTORY INDUCTION MECHANISM (AIM) TO THE EXTRAPOLATION OF CHAOTIC TIME SERIES
}

\author{
Dennis S. Buck \\ Dale E. Nelson \\ Avionics Directorate \\ Wright Laboratory \\ Wright-Patterson Air Force Base, OH 45433-6543 \\ buckds@wl.wpafb.af.mil nelsonde@wl.wpafb.af.mil
}

\begin{abstract}
This paper presents research done as part of a large effort to develop ontogenic (topology synthesizing) neural networks. One commerically available product, considered an ontogenic neural network, is the Abductory Induction Mechanism (AIM) program from AbTech Corporation of Charlottesville, Virginia. AIM creates a polynomial neural network of the third order during training. The methodology will discard any inputs it finds having a low relevance to predicting the training output. The depth and complexity of the network is controlled by a user-set Complexity Penalty Multiplier (CPM). This paper presents results of using AIM to predict the output of the Mackey-Glass equation. Comparisons are made based on the RMS error for an iterated prediction of 100 time steps beyond the training set. The data set was developed using a Tau value of 17 which yields a correlation dimension (an approximation of the fractal dimension) of 2.1. We explored the effect of different CPM values and found that a CPM value of 4.8 gives the best predictive results with the least computational complexity. We also conducted experiments using 2 to 10 inputs and 1 to 3 outputs. We found that AIM chose to use only 2 or 3 inputs, due to its ability to eliminate unnecessary inputs. This leads to the conclusion that Takens' theorem cannot be experimentally verified by this methodology! Our experiments showed that using 2 or 3 outputs, thus forcing the network to learn the first and second derivative of the equation, produced the best predictive results. We also discovered that the final network produced a predictive RMS error lower than the Cascade Correlation method with far less computational time.
\end{abstract}

\section{INTRODUCTION}

This work is part of a larger project entitled "Ontogenic Neural Networks for the Prediction of Chaotic Time Series". The American Heritage Dictionary of the English Language defines the term ontogenic as "the course of development of an individual organism", which comes from the biological term ontogenesis. Thus, ontogenic neural networks evolve their structures as they determine their own (hopefully) optimum topology during training. Conventionally, most researchers try to find an optimum topology by using "rules of thumb" to determine an initial network topology and then use trial and error to find the best topology for their application. Thus, it was the researcher who determined the best topology through a very tedious and time consuming process. A process which was not guaranteed to produce the optimum topology. Most researchers really want neural networks to create their own topology, automatically. This has lead, in recent years to the development of ontogenic technology, algorithmic methods to give neural networks the capability to learn their own topology. Further, we are gradually working to develop paradigms that endeavor to determine the optimum network architecture.

In this study we are trying to predict future values of a chaotic function. Chaos is best described as "... the complicated behavior of simple deterministic equations. The hallmark of a chaotic system is the sensitivity of the system's individual trajectories to their initial conditions... Long-term predictions of chaotic systems are virtually impossible. Even if the physics of a chaotic system is known completely, errors in measuring the initial state propagate exponentially." [7] We are seeking to predict the future $20 \%$ beyond our training data set.

One baseline ontogenic methodology for this study is the Abductory Induction Mechanism (AIM). This paper presents some of the work done using the AIM methodology which was developed by AbTech Corporation of Charlottesville, Virginia. AIM is an advanced machine learning tool that automatically discovers network solutions to complex decision, prediction, control, and classification problems. Given a database of examples, AIM synthesizes abductive networks and encodes them into computer subroutines that can be readily incorporated into application software. Abductive networks can accurately capture complex and subtle relationships that cannot be practically modeled using current symbolic or statistical methods. AIM provides a complete environment to synthesize, analyze, and encode abductive networks [2].

\section{DATA}

For this study, the Mackey-Glass equation was chosen as the generator of the chaotic time series. This equation has been studied by many authors such as Lapedes and Farber [5] and 
Stright [6]. The equation is a nonlinear differential delay equation:

$$
\frac{d x(t)}{d t}=\frac{a x(t-\tau)}{1+x^{10}(t-\tau)}-b x(t)
$$

The parameter $t$ is the time variable, $x$ is a function of $t$ whereas $\mathrm{a}, \mathrm{b}$, and $\tau$ (tau) are constants. The constant values $a$ and $b$ are 0.2 and 0.1 respectively. Tau $(\tau)$ is the time delay which can be used to vary the degree of chaos in the system (we chose $\tau=17$ which is just beyond the on-set of chaos). We solved the Mackey-Glass equation using a predictor-corrector numerical method [6] to determine the $\mathrm{x}$ values. The data set used in this experiment consists of values of $x$ computed at equal time intervals of 6 .

Observing the behavior of a chaotic system may lead to the belief that the underlying mathematics are quite complex. However, the Mackey-Glass function, graphed in Figure 1, can be described by a relatively simple deterministic equation. This leads us to the hope that we might be able to reliably predict the future values of this system. In essence, the network is trying to become a model of the underlying system.

The use of $\tau=17$ is based on previous work by Lapedes and Farber [5] and investigated by Gearhart [4]. As $\tau$ increases, chaos in the system increases. The amount of chaos is measured by the Correlation Dimension which is 2.1 , for this case.

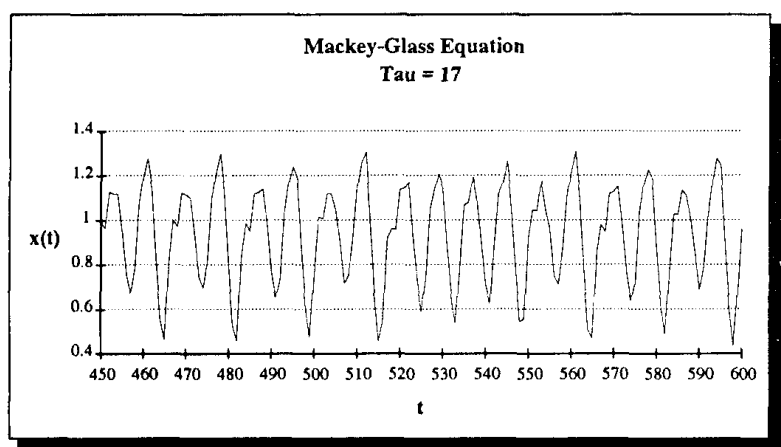

Figure 1

\section{METHOD OF EXTRAPOLATION}

Extrapolation is an attempt to predict values of a data series that lie beyond the end of the series. This is different from interpolation where values between known values are being approximated. Basically there are two ways to extrapolate a time series using neural networks. If it is desired to predict a value $\Delta t$ beyond the known series, the network is trained on data $\Delta t$ apart. When the last known values of the series are input, the network will output the desired values $\Delta t$ in the future. The second approach is to train the network on data $\delta t$ apart where $\delta t \ll \Delta t$. The outputs of the network are fed back to the network as inputs until the desired number of predictions have been made, $(\Delta t / \delta t)$. This approach is known as iterated prediction. In order to make this process very clear we will present a detailed discussion of how this is done. For an $\mathrm{n}$ input, $\mathrm{m}$ output network, a training exemplar is created by taking $n+m$ consecutive values from the data set to be predicted. Starting at an initial value $x_{j}$, the consecutive $n$ values of input are presented as $\left(x_{i}, \ldots, x_{n+i-1}\right)$ and the successive output $m$ values for these $n$ values of input are $\left(x_{n+1}, \ldots\right.$, $\left.x_{n+m+i-1}\right)$ for calculating the training error. Hence, $\left(x_{i}, \ldots\right.$, $\left.x_{n+i-1}, x_{n+1}, \ldots, x_{n+m+i-1}\right)$ can be shown as a general training exemplar for an $n$ input, $m$ output network.

Let the last known value in the data set be $x_{k}$. Let $p_{1}$ be the first value to be extrapolated. In order to have consistent comparisons, we chose $k=450$, therefore, $p_{1}$ is the next value of $x$ beyond 450 . In order to distinguish the predicted values from the actual values, the predicted values are denoted as $p_{i}$ and actual values are denoted as $x_{i}$. Table 1 is an example of this process of iterated prediction for $n=4, m$ $=1$ and $\mathrm{k}=450$.

After the network is trained, the extrapolation (prediction) process begins, with the first predicted value, $p_{1}$ using the last $n$ values in the data set $\left(x_{k-n+1}, \ldots, x_{k}\right)$ as the network inputs. Thus for $p_{1}$, all inputs are known data values. The second prediction $p_{2}$ is created by presenting $\left(x_{k-1+2}, \ldots, x_{k}, p_{1}\right)$ to the network inputs. The subsequent input exemplars are shifted by one for following predictions. For $\mathrm{p}_{2}$, one input is an extrapolated value, for $\mathrm{p}_{3}$ two inputs are extrapolated values, and so forth, until finally, predictions are made based only upon previously predicted data.

\begin{tabular}{|c|c|c|c|c|}
\hline \multicolumn{4}{|c|}{ Neural Network Input } & NN Output \\
\hline$:$ & : & $:$ & : & $:$ \\
\hline$x_{446}$ & $X_{447}$ & $x_{448}$ & $x_{449}$ & $x_{450}$ \\
\hline$x_{447}$ & $\mathrm{x}_{448}$ & $x_{449}$ & $x_{450}$ & $\mathrm{p}_{1}$ \\
\hline$x_{448}$ & $\mathbf{x}_{449}$ & $\mathrm{x}_{450}$ & $\mathrm{p}_{1}$ & $\mathrm{p}_{2}$ \\
\hline$x_{449}$ & $\mathrm{x}_{450}$ & $\mathrm{p}_{1}$ & $\mathrm{p}_{2}$ & $\mathrm{p}_{3}$ \\
\hline$x_{450}$ & $\mathrm{p}_{1}$ & $\mathrm{P}_{2}$ & $\mathrm{p}_{3}$ & $\mathrm{p}_{4}$ \\
\hline$P_{1}$ & $\mathrm{P}_{2}$ & $\mathrm{P}_{3}$ & $\mathrm{P}_{4}$ & $P_{5}$ \\
\hline : & $:$ & : & $:$ & $:$ \\
\hline
\end{tabular}

Table 1 
$\underline{\text { AIM }}$

AIM is an advanced machine learning tool based upon abductive modeling. This methodology uses mathematical models to represent complex and uncertain relationships along with networks to represent the underlying process. AIM networks are feed-forward polynomial networks. AIM constructs a network of nodes which can be of several different types. Three of the types, Singles, Doubles, and Triples are third order polynomials with one, two or three inputs, respectively. For example, a Triple is defined by the equation:

$$
\begin{aligned}
\text { Output }= & w_{0}+w_{1} x_{1}+w_{2} x_{2}+w_{3} x_{3}+w_{4} x_{1}{ }^{2}+w_{5} x_{2}{ }^{2}+ \\
& w_{6} x_{3}{ }^{2}+w_{7} x_{1} x_{2}+w_{8} x_{1} x_{3}+w_{9} x_{2} x_{3}+ \\
& w_{10} x_{1} x_{2} x_{3}+w_{11} x_{1}{ }^{3}+w_{12} x_{2}{ }^{3}+w_{13} x_{3}{ }^{3}
\end{aligned}
$$

where $x_{1}, x_{2}, x_{3}$ are inputs and $w_{n}$ are the trained weights. Another kind of node used by AIM in these experiments is White. A White node is defined as:

$$
\text { Output }=w_{0}+w_{1} x_{1}+w_{2} x_{2}+\ldots+w_{n} x_{n}
$$

AIM uses two other kinds of nodes for the input and output layers which are Normalizers and Unitizers, respectively. These are defined as:

\section{NORMALIZER OUTPUT $=w_{0}+w_{1} x_{1}$ UNITIZER OUTPUT $=\mathrm{w}_{0}+\mathrm{w}_{1} \mathrm{x}_{1}$}

Normalizers transform the original input variables into a mean of zero and a variance of one using mean-sigma normalization. A Unitizer converts the range of network outputs to a range with the mean and variance of the output values used to train the network. [1]

A schematic example of an AIM network is shown in Figure 2. The operation of AIM does not necessarily produce an optimum result, however, AIM will capture complex and subtle relationships accurately.

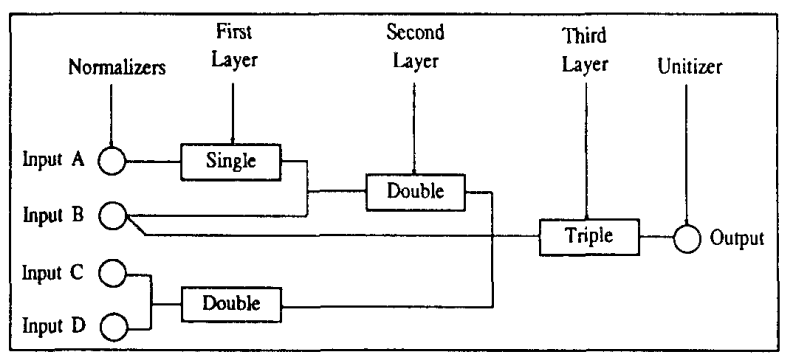

Example of AIM network

Figure 2

The Complexity Penalty Multiplier (CPM) is an AIM variable which controls the complexity of the network. The
CPM modeling criterion has been demonstrated to allow AIM to synthesize abductive networks with minimal user intervention. The CPM adjusts the trade-off between network complexity and modeling accuracy. This trade-off assumes that the simplest model that best fits the data will perform best on unseen data. As CPM increases, the network will be penalized more for complexity which encourages a simpler network. As CPM decreases, AIM will allow more complex networks which may over-fit the data. Adjusting the CPM influences the ability of the network to generalize and the computational time.

\section{EXPERIMENTS}

We performed three experiments which paralleled our study of the Cascade Correlation methodology. First we experimented to determine the best setting of the CPM value in the AIM program for our particular application. Second, we attempted to verify Takens' Theorem, experimentally, by varying the number of inputs in the training exemplars. The third experiment was to see if training on more output values would provide better predictions.

Since the value of CPM is the main control that the user has to determine the network complexity, our first experiments were to determine the best value of CPM for our problem. We varied CPM from 0.01 (the minimum permitted by the program) to 5.00 in an effort to determine the optimum setting for other experiments. In keeping with our experiments using the Cascade Correlation and following the lead of Lapedes and Farber [5], we chose four inputs and one output to observe how the predictive RMS varies with respect to the CPM value. Figure 3 shows the results of this experiment.

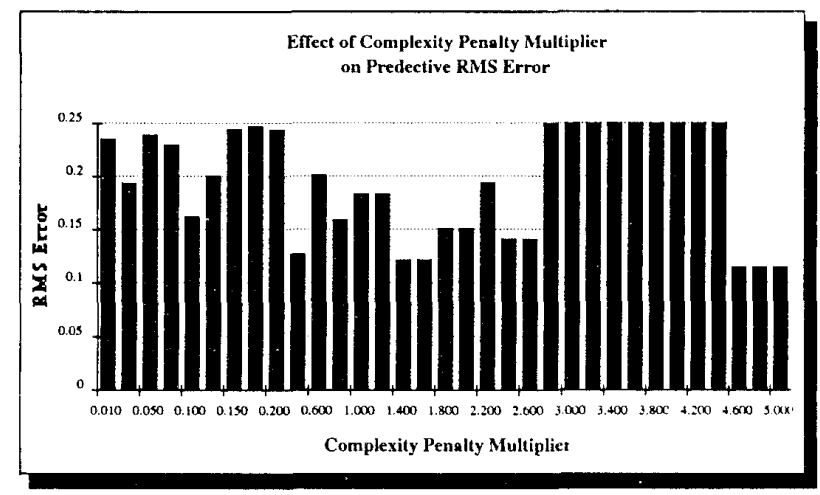

Figure 3

According to the bar chart, the minimum RMS Error occurs at CPM values of 4.6, 4.8 and 5.0, which have equivalent errors. We also notice that there is a roughly equivalent predictive error with a CPM value of 0.4 . As Figure 3 shows, there is no smooth pattern which will allow the user to select some minimum value. Figure 4 shows the network 
generated using a CPM of 0.4 and Figure 5 shows the network generated with a CPM value of 5.0. Since there is decreased computational time with larger CPM values, as shown in Figure 6, we decided to choose a larger CPM value which would also produce low predictive RMS results.

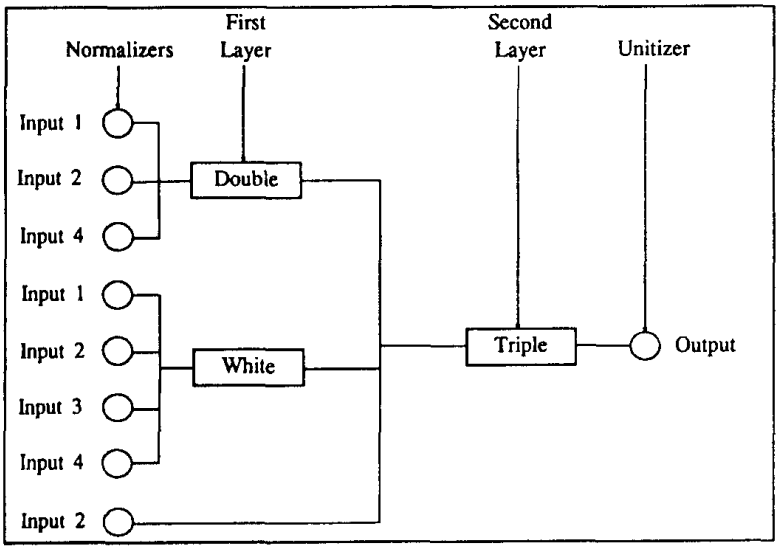

Network with CPM $=0.4$ Figure 4

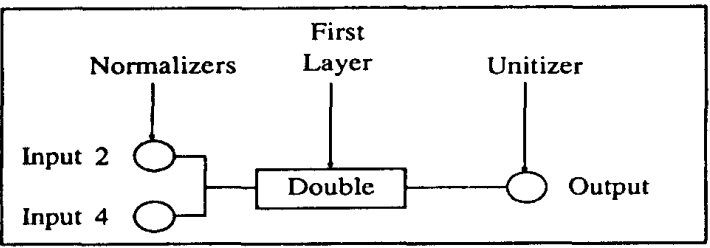

Network with $\mathrm{CPM}=5.0$

Figure 5

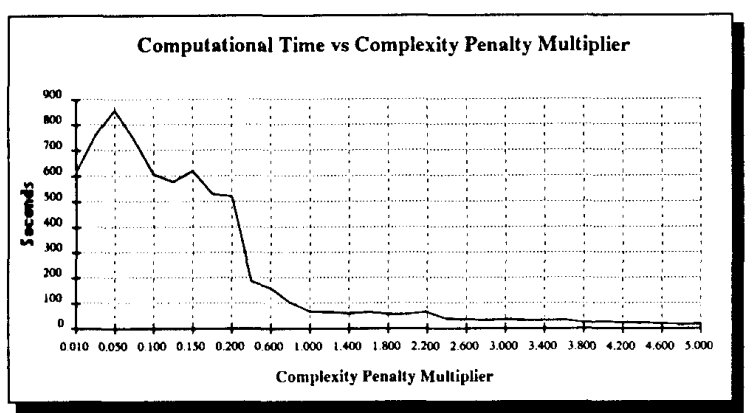

Figure 6

Based on the above results, a CPM value of 4.8 was used as the basis for our other experiments.

\section{Takens' Theorem}

Takens' theorem states that a least upper bound exists for which the function approximated by the neural network will be a smooth map. If the correlation dimension (an approximation of the fractal dimension) is defined to be $C_{d}$, then the required dimension $(n+1)$ must be less than or equal to $2 \mathrm{C}_{\mathrm{d}}+1$. Furthermore, the minimum dimension must be greater than or equal to $\mathrm{C}_{\mathrm{d}}$. Therefore, Takens' theorem may be stated as :

$$
\mathrm{C}_{\mathrm{d}} \leq n+1 \leq 2 \mathrm{C}_{\mathrm{d}}+1
$$

For the Mackey-Glass equation with $\tau=17\left(C_{d}=2.1\right)$, this would reduce to:

\section{$2.1 \leq n+1 \leq 5.2$}

Since the number of inputs to the network must be an integer, its value $(n+1)$ must be 3,4 , or 5 to satisfy the inequality.

To investigate the effect of the number of inputs and outputs on extrapolation accuracy, we trained networks with every combination of 2 through 10 inputs and 1 through 3 outputs. The two-output case forces the network to learn the first derivative (slope) of the function. The three-output case requires the network to, additionally, learn the second derivative (curvature) of the function. The second and third outputs are used for training purposes only and are ignored during extrapolation. Figure 7 shows the combination of provided inputs and outputs and the resulting RMS Error. Since AIM uses only the inputs it feels are important, we were unable to produce a graph showing the true effect of varying the number of inputs as was reported in our previous publication [3]. If we had chosen a lower CPM value, the networks generated would have chosen to use more of the inputs. See Figure 4. However, we felt that the additional time required and the increased computational complexity would not provide us with much better results in the verification process. AIM's results provide more evidence to support the claim that the lower bound of Takens' theorem is the correct number of inputs to use. We will attempt to verify this observation through experiments using data of higher correlation dimension.

\section{Effect of Number of Inputs and Outputs}

In this study, AIM only chose to use two or three inputs to predict future output even though a larger number of inputs have been provided. The process in AIM determines the number of necessary inputs and which ones they should be.

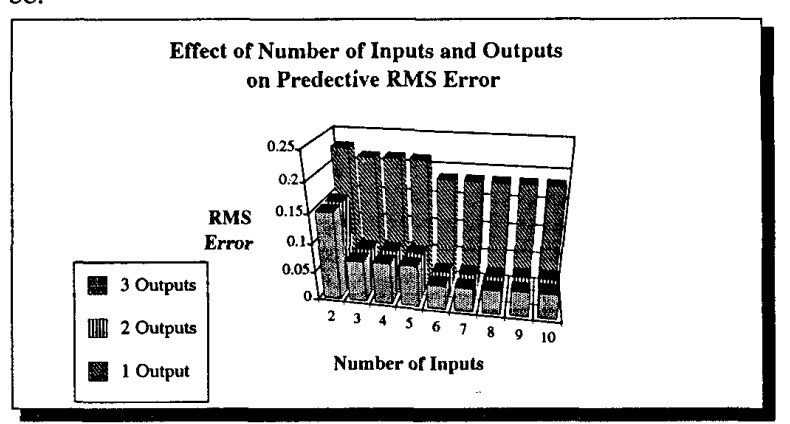

Figure 7 
Table 3 shows the total number of inputs presented, which input elements AIM chose to use from the inputs available, number of inputs used for the AIM network, number of outputs presented, and the predictive RMS. There are several interesting results seen in Table 3. First, is that the predictive RMS error is the same whether there are two or three outputs. Therefore, forcing the network to learn the second derivative does not improve performance, at least for data with a correlation dimension of 2.1. This may not be the case with higher dimensional data. Second, AIM only chooses the most recent data points and usually not two consecutive points for prediction. Three points were only chosen when the network was forced to learn both first and second derivatives. It is interesting that the network never chose to use more than three inputs and then only the most recent three. The minimum predictive error was achieved when using two inputs and two outputs.

\begin{tabular}{|c|c|c|c|c|}
\hline Inputs Applied & Inputs Used & \# Inputs Used & \# Outputs & RMS Error \\
\hline 2 & 1,2 & 2 & 1 & 0.226876 \\
\hline 2 & 1,2 & 2 & 2 & 0.151437 \\
\hline 2 & 1,2 & 2 & 3 & 0.151437 \\
\hline 3 & 1,3 & 2 & 1 & 0.213215 \\
\hline 3 & 1,3 & 2 & 2 & 0.071744 \\
\hline 3 & 1,3 & 2 & 3 & 0.071744 \\
\hline 4 & 2,4 & 2 & 1 & 0.213215 \\
\hline 4 & 2,4 & 2 & 2 & 0.071744 \\
\hline 4 & 2,4 & 2 & 3 & 0.071744 \\
\hline 5 & 3,5 & 2 & 1 & 0.213215 \\
\hline 5 & 3,5 & 2 & 2 & 0.071744 \\
\hline 5 & 3,5 & 2 & 3 & 0.071744 \\
\hline 6 & 4,6 & 2 & 1 & 0.182663 \\
\hline 6 & 4,6 & 2 & 2 & 0.041403 \\
\hline 6 & $4,5,6$ & 3 & 3 & 0.041403 \\
\hline 7 & 5,7 & 2 & 1 & 0.182663 \\
\hline 7 & 5,7 & 2 & 2 & 0.041403 \\
\hline 7 & $5,6,7$ & 3 & 3 & 0.041403 \\
\hline 8 & 6,8 & 2 & 1 & 0.182663 \\
\hline 8 & 6,8 & 2 & 2 & 0.041403 \\
\hline 8 & $6,7,8$ & 3 & 3 & 0.041403 \\
\hline 9 & 7,9 & 2 & 1 & 0.182663 \\
\hline 9 & $7,8,9$ & 3 & 2 & 0.044311 \\
\hline 9 & $7,8,9$ & 3 & 3 & 0.044311 \\
\hline 10 & 8,10 & 2 & 1 & 0.182663 \\
\hline 10 & $8,9,10$ & 3 & 2 & 0.044311 \\
\hline 10 & $8,9,10$ & 3 & 3 & 0.044311 \\
\hline
\end{tabular}

Table 3

\section{CASCADE CORRELATION COMPARISON}

The lowest predictive RMS value obtained with AIM was 0.041403. Similar experiments with Cascade Correlation produced a predictive RMS value of 0.172 . However, the results produced by Cascade Correlation would depend upon the random starting seed and required considerably more time to generate a network. AIM produces the same results, given the same CPM value and data set for training. Further, AIM produces the result in much less time, usually orders of magnitude less time. Another problem which would sometimes occur in Cascade Correlation is that even though the training was the same for all runs, occasionally we would see predictive values go to infinity within $10 \Delta \mathrm{t}$. A similar result was never seen using AIM.

\section{CONCLUSIONS}

AIM appears to be a very robust, easy to use system capable of modeling chaotic time series of low dimensionality. It may be able to handle higher dimension cases, but, this need to be verified. AIM produces results which are better than Cascade Correlation with much less time and effort. Further, the models produced are much less computationally complex.

We were unable to produce a smooth curve to show whether Takens' Theorem can be proven experimentally using the AIM methodology. AIM provides evidence that the lower bound of the Takens' inequality, for low dimensional data, is correct.

We have shown that, for prediction, the use of two outputs to force the network to learn the first derivative of the underlying function will greatly reduce the predictive RMS error. We have also shown that there is apparently no benefit to having the network learn the second derivative of the underlying function. Both of these conclusions are made for the AIM methodology and for this particular set of data. More experiments must be accomplished to determine whether this will hold for other methodologies and higher dimensional data..

\section{FUTURE WORK}

We are attempting to find/develop a methodology which will be able to predict chaotic time series far into the future. We have chosen to benchmark the effort with AIM, an ontogenic polynomial network paradigm, Cascade Correlation, a non-polynomial ontogenic paradigm, and back propagation. We are expanding our experiments in all three areas to include data of higher correlation dimension. We will also be examining recent developments in the Cascade Correlation paradigm; Recurrent Cascade Correlation, Cascade II, and Recurrent Cascade II. 
We thank Keith Drake from AbTech Corporation for his ultimate effort to support our work.

\section{REFERENCES}

[1] AbTech Corporation, AIM User's Manual, 1991.

[2] Drake, K., "Abductory Induction Mechanism (AIM)", AbTech Corporation, 1989.

[3] Ensley, D., Nelson D., "Applying Cascade Correlation to the Extrapolation of Chaotic Time Series", Proceeding of the Third Workshop on Neural Networks: Academic/Industrial/ NASA/Defense 10-12 Feb 1992.

[4] Gearhart, T., "Correlation Dimension of Chaotic Attractors", Capitol University, Columbus Ohio, 1991.

[5] Lapedes, A., Farber, R., "Nonlinear Signal Processing Using Neural Networks: Prediction and System Modeling", Los Alamos National Laboratory LA-UR-87-2662, June 1987.

[6] Stright, J., "A Neural Network Implementation of Chaotic Time Series Prediction", Air Force Institute of Technology, AFIT/GE/ENG/88D-50, December 1988.

[7] Theiler, J., "Estimating the Fractal Dimension of Chaotic Time Series", The Lincoln Laboratory Journal, Volume 3, Number 1, 1990. 